\title{
Emotional Personality Traits and Their Relationship in Adolescents with Chronic Physical IIIness
}

\author{
Larisa B. Dykhan \\ Southern Federal University \\ Ludmila V. Voskovskaya \\ Southern Federal University \\ Valentina V. Pizhugiyda \\ Southern Federal University \\ Natalia N. Malyarchuk \\ Tyumen State University \\ Natalia V. Semyenova \\ Tyumen State University \\ Email: eldirect@mail.ru,dyhanlb@mail.ru
}

\section{Doi:10.5901/mjss.2015.v6n3s2p269}

\section{Abstract}

Currently, we observe an increase in the growth rate of chronic diseases among adolescents. Somatic diseases are everywhere recognized as conditioned by emotional problems among other factors. Often the researches explore the emotional patterns that are typical for a particular nosology. The purpose of this study is to identify general emotional personality traits in adolescents with different chronic physical illnesses of visceral organs. Neuroticism, trait anxiety, emotional tension, mental instability, sensitivity, aggressive reactions have been studied using following techniques: EPQ, STAI, BDHI, "Accumulation of emotional charges aimed at himself" test, multivariate personality questionnaire "Psychodiagnostic test." In contrast to healthy peers, chronically ill adolescents have higher levels of trait anxiety and mental imbalance, higher levels of neuroticism and irritability. Levels of emotional tension, indirect aggression, tendency to depression and sensitivity are slightly higher, the differences are minimal. We have observed much more extensive relations of neuroticism, psychoticism and irritability with other traits in adolescents with chronic physical illnesses. Correlations of emotional tension (as a habit to suppress emotions) are limited only by intrapersonal problems "psikhotizm", hostility and guilt. A sort of «blind spot» have been observed: a lack of significant relations of emotional tension with trait anxiety, neuroticism and a tendency to depression, which often can be considered as predictors of chronic physical illnesses. We have supposed, that teenagers with chronic physical illnesses are not aware of some part of their emotional tension, and this phenomenon may be a factor of chronically supported somatization.

Keywords: chronic physical illness, emotional sphere, adolescents;

\section{Introduction and Literature Review}

Scientific investigations and the statistics of the Russian health care system show an increase in the number of children with chronic diseases, especially among adolescents of 14 to 17 years. These adolescents have trouble adapting to society more often than their healthy peers. The disease leads to the disability and causes social failure in adult life (A.A. Baranov, 2012). A similar trend has been also observed in the United States (J.V. Perrin et al., DOl:10.1001/jama.297. 24.2755, 2007; N. Halfon and P.W. Newacheck, DOI:10.1001/jama.2010.130, 2010).

Among all the chronic diseases there is a special group of psychosomatic disorders, where a significant role in the appearance and course is played by psychological factors (N.N. Malyarchuk, 2010). Study of personality characteristics of adolescents with chronic diseases conducted in Russia by V. Nikolaeva, E. Sokolova, T. Goriacheva, N. A Kovalenko 
and others in 80-90th of the last century (I.G., Malkina-Pih, 2008), the work of S. Weiland, S. Power, J. Lavigne and others (J.C. Suris, DOI:10.1136/adc.2003.045369, 2004) show patterns typical for a particular nosology. Recent research studies confirm and refine these patterns. For example, children and adolescents with diseases of the gastrointestinal tract have a high level of trait anxiety, unmet need for love and recognition, self-centeredness, the simultaneous desire to support and lead, difficulty in relationships with adults and peers (I.A. Kazakov, 2009). Adolescents with hypertension are characterized by high anxiety coupled with emotional lability, isolation, stress-demanding attitude to life (Long et al., 2010). Children with asthma are recorded with increased responsibility and commitment to social norms, a tendency to curb their emotional reactions and its increased cognitive control, care, dependence on other people's opinions.

The risk of psychosomatic diseases is traditionally associated with a long uncontrolled emotional stress (G.I. Efremova et al., DOl:10.5539/ass.v10n22p263 2014). L.P. Marincheva et al. believe that the pathogenesis of psychosomatic pathology is multi-factor. Psychosomatic illness, in their opinion, is a non-specific response to stress. Its development stages begin from the symptoms somatomorphic functional deviations and end by the development of the true psychosomatics. The authors considered the personality characteristics of adolescents as one of the most important factors in the pathogenesis (L.P. Marincheva et al., 2012).

A.B. Kholmogorova and N.G. Garanyan focus on a relationship between the psychosomatic diseases and culturally determined ways of emotional response. The authors show that "the cult of success" widespread in any society (due to the retroactivity effect of overvalued aim) leads to the development of depressive symptoms, "the cult of force" - to the increase in anxiety, "the cult of rationality" - to the somatization due to unresolved emotional issues (A.B. Kholmogorova and N.G. Garanyan, 1999). Cultural traditions of expressing emotions affect the formation of adolescents' personality and, respectively, their physical health. It may impose restrictions on the cross-cultural transfer of findings obtained on the empirical data in different countries.

Works addressing personal characteristics typical for chronically ill children and adolescents are either comparative empirical studies of two or three nosologies, or reviews and meta-analyzes, in which there is a total unification of chronic diseases, including cancer. Most authors have recorded an increased trait anxiety among chronically ill children and adolescents (Y.E. Kurtanova, http://psychlib.ru/inc/absid.php?absid=78515, 2004; M. Pao and A. Bosk, DOI:10.1002/ da.20727, 2011 et al.). They have mentioned such characteristics as chronic emotional stress, latent or open aggression, susceptibility to depression, self-doubt, isolation, dependence on other people's opinions (O.V. Frolov, 2009; M. Pinquart and Y. Shen, DOI:10.1093/jpepsy/jsq104, 2011; M. Pinquart and Y. Shen, DOI:10.1093/jpepsy/jsr042, 2011;), as well as lower levels of self-esteem and its more rapid decrease in adolescence (M.A. Ferro and M.H. Boyle, DOI:10.1093/jpepsy /jss112, 2013). Taken together, these patterns are described in the foreign studies as internalization symptoms (Suris et al., 2004). It has been noted that the most part of the studies on the emotional sphere of adolescents with chronic physical disorders has been performed within nosocentric paradigm, and there is a lack of proper psychological research (I.A. Kazakov, 2009; Pinquart and Shen, 2011).

Thus, the search for common emotional personality traits of adolescents with chronic physical diseases is far from being complete explaining our interest to the problem.

The purpose of our empirical study is to identify common emotional personality traits and their relationships among the adolescents with chronic physical illness. The diseases of visceral organs have been chosen, the psychosomatic origin of which is recognized by most scientists. We have studied neuroticism, trait anxiety, emotional tension, different aggressive reactions, mental instability and sensitivity.

\section{Research Methodology}

\subsection{Participants}

The study involved 60 pupils of the Rostov region (Russia) of 15-16 years old (29 boys and 31 girls). The survey has been conducted with the consent of teenagers and their parents in an anonymous form. The experimental group (EG) included 30 adolescents with a chronic physical illness (14 boys and 16 girls). According to their medical records there were 9 persons with a disease of gastrointestinal tract, 9 persons - of cardiovascular system, 5 persons - of airway and 7 persons - of kidney. Those teens during the previous two years were treated in hospital and were observed by a doctor. At the time of the survey, they were going to school; their disease was in a stage of relative remission. The control group (CG) consisted of 15 boys and 15 girls. According to their medical records they were in a good health, which was confirmed by a personal conversation. 


\subsection{Instruments}

Emotional and personal characteristics of adolescents have been determined using a number of techniques.

Emotional stability / instability has been determined using Neuroticism / Stability Scale (N-Scale) of Eysenck Personality Questionnaire (EPQ), completed with a secrecy-frankness scale. Russian version was adapted by A.G. Shmelev (A.G. Shmelev, 1988). The questionnaire consists of 33 "yes / no" questions. The maximum score is 24. Emotional stability is fixed at 12 points or less, evident emotional instability - at 19 points or greater.

Trait Anxiety (TA) has been studied using State-Trait Anxiety Inventory (STAI) by Spielberger et al. Russian version was adapted by Y.L. Khanin (Y.L. Khanin, 1976). We have used an trait anxiety scale including 20 questions and answers in the range from 1 to $4 \mathrm{~b}$. The maximum score is 80 . A score of less of equal to 30 points corresponds to low anxiety, of 31-44 points - moderate, of 45 or more - high anxiety.

Emotional tension (ET) has been evaluated by the test "Accumulation of emotional energy charges aimed at himself» (AECAH) of $\mathrm{V}$. Boyko. We used its modification of $\mathrm{E}$. llyin allowing estimating the negative emotions that do not receive outside exit (E.P. Ilyin, 2002). The statements of the questionnaire relate to a tension resulting from the emotions that are constrained and do not appear outside. Objects of suppressed emotions are the man himself, his environment, as well as the circumstances of life. In fact, the questionnaire records the habit of suppressing negative emotions (anger, resentment, fear).

Mental instability and sensitivity have been investigated by the multi-factor questionnaire called "Psychodiagnostic test» (PDT), developed by V. Melnikov and L. Yampolsky (V.M. Melnikov and L.T. Yampolsky, 1985). The questionnaire is aimed at studying the structure of personality within the boundaries of certain factors. It contains 174 short "true / false" statements. Questionnaire items are grouped into 10 elementary subscales, as well as 4 generalizing scales (factors). The presented study has recorded the results for only two factors associated with the emotional sphere of personality. The scale "Mental Instability» (MI) includes subscales "Neuroticism" (Neur), "Psychoticism" (Ps) and "Depression" (Dep). The Scale "Sensitivity" (Sen) includes subscales "Aesthetic sensibility" (AeS) and "Femininity" (Fem). The results for the scales (factors) are calculated not by summing the subscale values, but by summing of a number of its questions having the greatest factor loadings. Initial estimates have been converted into walls following the rules defined by the test results for 1315 adolescents of 15-17 years old (L.A. Pergamenschikov et al., 1992).

Aggression and hostility have been determined by the Buss-Durkee Hostility Inventory (BDHI). We have used the Russian version of this methodology, adapted by S.N. Yenikolopov (S.N. Yenikolopov, 1990). It consists of 75 "yes/no" questions. Items of the questionnaire are in 8 subscales: 1) Assault (Acc), 2) Indirect aggression (Ind), 3) Irritability (Irr), 4) Negativism (Neg), 5) Resentment (Res), 6) Suspiciosness (Sus), 7) Verbal aggression (Ver), 8) Guilt (Guilt). Initial estimates are converted into walls. Index of hostility $(\mathrm{IH})$ is defined as the sum of values over the subscales 5 and 6 . The index of aggressiveness $(I A)$ is the sum of values over the subscales $1,2,7$. The evaluation rate of the index of aggressiveness is $21 \pm 4$, the one of the index of hostility is $6.57 \pm 3$.

Statistical analysis has been performed using STATISTICA (data analysis software system), version 10.0 for Windows, StatSoft, Inc. Expression level differences of a characteristic have been determined by $\mathrm{U}$ - Mann-Whitney test, since in some cases the distribution of a characteristic differed from normal. Correlations have been determined using the Spearman's rank correlation coefficient. Differences were considered statistically significant at $p<0.05$.

\section{Findings and Discussion}

\subsection{Intensity of the investigated emotional and personal characteristics}

The survey recorded a number of significant differences in the intensity of emotional personality traits of EG adolescents versus CG teenagers. The most significant differences are observed in the intensity of neuroticism and trait anxiety. Based on average indices we have recorded elevated levels of trait anxiety in EG adolescents against average TA level in CG adolescents. (Table. 1). 
Table 1. Medians, quartiles and the values of $U$ - Mann-Whitney test for the studied characteristics in adolescents with chronic physical illness (EG) and in healthy adolescents (CG)

\begin{tabular}{|cccccccccccc|}
\hline \multicolumn{2}{|c}{ Tests } & EPQ & STAI & AECAH & \multicolumn{1}{c|}{ PDT } \\
\hline \multicolumn{2}{|c}{ Indicators } & N-Sc & TA & ET & Neur & Ps & Dep & MI & AeS & Fem & Sen \\
\hline EG & Md & 16.5 & 47.5 & 9 & 7.5 & 5.5 & 6 & 7 & 5 & 6 & 5 \\
$n=30$ & $\mathbf{Q}_{1} \mathbf{Q}_{3}$ & $13-18.3$ & $42-54.3$ & $7-12.3$ & $6-9$ & $3.8-7$ & $4.8-8$ & $4-7.2$ & $4-6$ & $5-7$ & $3-6$ \\
\hline $\mathbf{C G}$ & $\mathbf{M d}$ & 11.5 & 40.5 & 8 & 5 & 5 & 4.5 & 5 & 4 & 5 & 3.5 \\
$\mathrm{n}=30$ & $\mathbf{Q}_{1}-\mathbf{Q}_{3}$ & $8.8-14.3$ & $34.8-45.5$ & $5-10.3$ & $3-6$ & $3-6$ & $3-7$ & $3-7$ & $3-5$ & $3.8-6.3$ & $3-6$ \\
\hline & $\mathbf{U}$ & 170.5 & 247 & 338 & 181.5 & 391 & 325.5 & $\mathbf{2 9 3}$ & $\mathbf{2 7 5 . 5}$ & $\mathbf{2 8 1 . 5}$ & 332.5 \\
& $\mathbf{P}$ & $<0.01$ & $<0.01$ & $=0.05$ & $<0.01$ & $>0.05$ & $<0.05$ & $<0.05$ & $<0.01$ & $<0.01$ & $<0.05$ \\
\hline
\end{tabular}

Note: $\bigcup_{0.05}=338 ; \bigcup_{0.01}=292$.

The average indicators for emotional instability (Neuroticism) in EG adolescents approach to high levels, while in CG adolescents that index is low. Neuroticism data obtained using the PDT procedure, show consistent results, but demonstrate slightly greater shift toward apparent neurotism in both groups. Markedly less pronounced, but significant differences between the groups are observed on the propensity to depression. Propensity to depression tendency (PDT) in EG adolescents is greater, it is close to the upper boundary of the average level, but the differences are small compared to CG. Neuroticism is defined as a predisposition to frequent and intense experience of a wide range of negative emotions. It is considered a more common characteristic with respect to such negative phenomena as anger, anxiety and depression (M.B. Howren and J. Suls, DOI:10.1037/a0021715, 2011). That is why, probably, as discussed before in the literature review, these characteristics often act as a general predictor of chronic somatic diseases. Our data thus consistent with results of previous studies.

Recall that in addition to the propensity to depression tendency and neuroticism, the scale "mental instability" includes a subscale called "psychoticism". For this indicator, there are no significant differences between EG and CG, its medians are found within the boundaries of the average. The differences over the «mental instability» scale are quite pronounced (see. Table. 1). The median of this indicator in EG teens is found at the lower boundary of the high level, significantly greater than in healthy adolescents. According to the interpretation of the authors of the methodology, we can assume a mental unbalance in chronically ill adolescents, understood as maladjustment, anxiety, loss of control over impulses. The subscales' and the "sensitivity" scale indicators in EG teenagers compared to the CG are also significantly higher. Chronically ill adolescents are more sensitive, vulnerable, delicate and modest.

Emotional tension as a habit to suppress negative emotions in EG adolescents is greater, but the differences are minimal, almost negligible (see. Table $1, p=0.05$ ). In both groups, the medians are at the average level of intensity of the characteristic. Thus, at first glance, EG teenagers do not have expressed concerns related to the habit of suppressing negative emotions.

Thus, the above results fit into the concept of «internalization symptoms», adopted in foreign psychology and the most frequently cited as the main difference between adolescents with chronic physical illnesses. In Russian works, such acts are often described as maladjustment to the asthenic type.

The study of different forms of aggressive reactions in teens shows a significant similarity between the groups (Table. 2).

Table 2. Medians, quartiles and values of $U$ - Mann-Whitney test on scales of the BDHI methodology in adolescents with chronic physical illness (EG) and in healthy adolescents (CG)

\begin{tabular}{|cccccccccccc|}
\hline \multicolumn{2}{|c}{ Indicators } & Acc & Ind & Irr & Neg & Res & Sus & Ver & Guilt & IH & IA \\
\hline EG & Md & 6 & 7 & 6 & 6 & 5 & 5 & 7 & 7 & 10 & 18.5 \\
$\mathrm{n}=30$ & $\mathbf{Q}_{1}-\mathrm{Q}_{3}$ & $4-7.3$ & $4-8$ & $4-7$ & $4-8$ & $4-6$ & $4-6$ & $5-8.3$ & $5-8$ & $9-13$ & $14.8-24$ \\
\hline $\mathbf{C G}$ & $\mathbf{M d}$ & 6 & 6 & 3 & 7 & 5.5 & 5 & 6 & 7 & 11 & 17.5 \\
$\mathrm{n}=30$ & $\mathbf{Q}_{1}-\mathrm{Q}_{3}$ & $3.8-8$ & $3-7$ & $1.8-6$ & $4-8$ & $3-7$ & $4-6.3$ & $4.8-8$ & $4-8.3$ & $7.8-12.3$ & $13-21.3$ \\
\hline & $\mathbf{U}$ & 436 & 335.5 & 259 & $\mathbf{3 8 5}$ & 424 & 398 & 387.5 & 404.5 & 404 & 367 \\
& $\mathbf{P}$ & $>0.05$ & $<0.05$ & $<0.01$ & $>0.05$ & $>0.05$ & $>0.05$ & $>0.05$ & $>0.05$ & $>0.05$ & $>0.05$ \\
\hline
\end{tabular}

Note: $U_{0.05}=338 ; \bigcup_{0.01}=292$

Medians over individual subscales in both groups for most cases are average; some of them are close to the upper bound. Average aggressiveness indicators approach to the lower bound of the average level. Hostility index indicators 
are high and reach the lower bound of the highest level. These results are consistent with the age characteristics of adolescence, stated in the literature (Xie et al., DOI:10.1002/ab.8000, 2002). Significant differences between the groups have been observed only for irritability and indirect aggression. Differences over the "irritability" subscale are significant. In EG adolescents, the median of this indicator is shifted to the upper half of the average level, but in CG adolescents - is at the low level $(p<0.01)$. In terms of indirect aggression, the differences are minimal and approach the negligibility bound $\left(\mathrm{U}_{\text {Ind }}=335.5, \mathrm{U}_{0.05}=338\right)$.

\subsection{Correlation analysis}

The results of the correlation analysis are shown in Table 3. The most significant correlations are fixed between the individual subscales and the scale, to which they belong. These facts are not discussed here because of their evidence. In the analysis, we will focus on the comparison of significant correlations $(p<0.05)$ observed between indicators in EG and $C G$ adolescents.

Table 3. Summary table of correlations between individual emotional and personal characteristics in adolescents with chronic physical illness (the upper part of the table, highlighted in gray) and their healthy peers (the lower part of the table)

\begin{tabular}{|c|c|c|c|c|c|c|c|c|c|c|c|c|c|c|c|c|c|c|c|c|}
\hline & N-Sc & TA & ET & Neur & Ps & Dep & MI & AeS & Fem & Sen & $\mathrm{IH}$ & IA & Acc & Ind & Irr & Neg & Res & Sus & Ver & Guilt \\
\hline $\mathrm{N}-\mathrm{Sc}$ & 1 & 0.72 & 0.25 & 0.51 & 0.38 & 0.28 & 0.55 & 0.49 & 0.39 & 0.38 & 0.43 & 0.27 & 0.1 & 0.31 & 0.41 & 0.14 & 0.31 & 0.32 & 0.31 & 0.13 \\
\hline TA & 0.35 & 1 & 0.29 & 0.6 & 0.41 & 0.41 & 0.58 & 0.34 & 0.35 & 0.3 & 0.66 & 0.3 & 0.16 & 0.41 & 0.65 & 0.04 & 0.45 & 0.48 & 0.21 & -0.05 \\
\hline ET & 0.39 & 0.58 & 1 & 0.25 & 0.52 & 0.3 & 0.49 & 0.01 & -0.19 & -0.31 & 0.42 & -0.08 & 0.05 & -0.08 & 0.3 & -0.14 & 0.21 & 0.35 & -0.16 & 0.44 \\
\hline Neur & 0.42 & 0.43 & 0.37 & 1 & 0.54 & 0.73 & 0.84 & 0.04 & 0.18 & 0.13 & 0.26 & 0.25 & 0.22 & 0.27 & 0.6 & 0.07 & 0.03 & 0.35 & 0.12 & -0.16 \\
\hline Ps & 0.16 & 0.33 & 0.46 & 0.46 & 1 & 0.59 & 0.68 & 0.04 & 0.06 & 0.13 & 0.45 & 0.39 & 0.43 & 0.27 & 0.59 & -0.05 & 0.29 & 0.37 & 0.31 & -0.03 \\
\hline Dep & 0.15 & 0.46 & 0.54 & 0.61 & 0.62 & 1 & 0.77 & 0.1 & 0.04 & 0.18 & 0.19 & 0.34 & 0.35 & 0.26 & 0.49 & 0.35 & -0.09 & 0.4 & 0.26 & -0.14 \\
\hline MI & 0.32 & 0.6 & 0.51 & 0.67 & 0.42 & 0.87 & 1 & 0.15 & 0.03 & 0.03 & 0.37 & 0.16 & 0.21 & 0.15 & 0.54 & 0.09 & -0.01 & 0.58 & 0.04 & -0.05 \\
\hline AeS & -0.31 & -0.03 & -0.17 & 0.03 & 0.04 & 0.24 & 0.04 & 1 & 0.27 & 0.62 & 0.19 & 0.05 & -0.13 & 0.06 & -0.1 & 0.33 & -0.12 & 0.41 & 0.25 & 0.12 \\
\hline Fem & 0.1 & 0.12 & 0.11 & 0.24 & 0.25 & 0.37 & 0.21 & 0.64 & 1 & 0.63 & 0.01 & 0.24 & -0.09 & 0.34 & 0.11 & -0.12 & 0.14 & -0.04 & 0.35 & -0.08 \\
\hline Sen & 0.14 & 0.01 & 0.05 & 0.2 & 0.08 & 0.24 & 0.17 & 0.72 & 0.77 & 1 & 0.01 & 0.3 & -0.01 & 0.39 & 0.13 & 0.18 & 0.03 & 0.05 & 0.42 & -0.35 \\
\hline IH & 0.14 & 0.59 & 0.34 & 0.43 & 0.28 & 0.28 & 0.37 & 0.14 & 0.05 & -0.01 & 1 & 0.33 & 0.29 & 0.39 & 0.68 & -0.03 & 0.72 & 0.66 & 0.23 & 0.09 \\
\hline IA & -0.24 & 0.2 & -0.15 & 0.35 & 0.21 & 0.25 & 0.28 & 0.44 & 0.17 & 0.29 & 0.41 & 1 & 0.88 & 0.88 & 0.44 & 0.48 & 0.43 & 0.02 & 0.83 & -0.28 \\
\hline Acc & -0.35 & -0.01 & -0.24 & 0.25 & 0.01 & 0.12 & 0.22 & 0.42 & 0.18 & 0.21 & 0.2 & 0.77 & 1 & 0.66 & 0.39 & 0.39 & 0.31 & 0.04 & 0.62 & -0.23 \\
\hline Ind & 0.19 & 0.48 & 0.13 & 0.37 & 0.28 & 0.37 & 0.45 & 0.16 & 0.04 & 0.09 & 0.53 & 0.7 & 0.3 & 1 & 0.49 & 0.33 & 0.49 & 0.037 & 0.6 & -0.37 \\
\hline Irr & -0.07 & 0.21 & -0.1 & 0.41 & 0.3 & 0.2 & 0.19 & 0.41 & 0.35 & 0.34 & 0.32 & 0.71 & 0.57 & 0.56 & 1 & -0.03 & 0.56 & 0.41 & 0.26 & -0.19 \\
\hline Neg & 0.2 & -0.1 & -0.04 & 0.11 & 0.13 & 0.08 & 0.05 & 0.01 & 0.21 & 0.17 & 0.11 & 0.17 & 0.01 & -0.03 & -0.07 & 1 & -0.14 & 0.11 & 0.56 & -0.07 \\
\hline Res & 0.2 & 0.51 & 0.36 & 0.33 & 0.24 & 0.27 & 0.32 & 0.16 & 0.07 & 0.06 & 0.85 & 0.42 & 0.14 & 0.57 & 0.23 & 0.19 & 1 & 0.03 & 0.33 & 0.04 \\
\hline Sus & 0.06 & 0.45 & 0.21 & 0.36 & 0.33 & 0.19 & 0.26 & 0.1 & 0.02 & -0.06 & 0.85 & 0.26 & 0.14 & 0.31 & 0.31 & 0.05 & 0.47 & 1 & 0.02 & 0.11 \\
\hline Ver & -0.32 & -0.07 & -0.19 & 0.17 & 0.22 & 0.05 & -0.08 & 0.36 & 0.14 & 0.32 & 0.19 & 0.67 & 0.34 & 0.18 & 0.44 & 0.38 & 0.22 & 0.13 & 1 & -0.1 \\
\hline Guilt & 0.33 & 0.73 & 0.54 & 0.25 & 0.23 & 0.44 & 0.52 & -0.03 & 0.11 & 0.06 & 0.5 & -0.07 & -0.18 & 0.22 & -0.08 & 0.01 & 0.49 & 0.37 & -0.22 & 1 \\
\hline
\end{tabular}

First of all, we have observed that in EG adolescents, there are extensive meaningful relations between Eysenck's neuroticism and the other characteristics, which are not typical for CG teenagers. The most pronounced relation is to trait anxiety ( $r_{S E G}=0.72$ ), which is absent in CG adolescents. These results seem natural to us, given that many of the indicators, especially neuroticism itself, are increased in adolescent patients, and neuroticism reflects the most common manifestations of negative affectivity.

Adolescent patients have also quite extensive relations to psychoticism. In addition to the (similar to CG) relations to the emotional tension, as well as with other subscales of the "mental instability" scale, in EG there are relations to the emotional instability (neuroticism), anxiety, irritation, to the indexes of hostility and aggression, and such their subscales as suspicion and physical aggression. Psychoticism according to existing methodologies reflects the closure, internal conflicts, unmotivated tension, excitement and affective "explosions". It can be assumed, that such extensive psychotism's relations say, that the EG adolescents are more likely to bring their intrapersonal problems to their perception of a variety of affective events that have been offered to them during the interview.

Trait anxiety is extensively related to other indicators in both groups. In correlation with mental instability, which can be called extensive in both groups, there is a difference only in relations to various aggressive reactions. In CG, it is due to the indirect aggression and guilt; in EG - to the irritation and suspicion. 
The analysis of relations of emotional tension as a habit to suppress negative emotions is of our particular interest. In healthy adolescents the emotional tension was significantly correlated with neuroticism, trait anxiety, mental instability and incorporated neuroticism, psychoticism, depression, as well as resentment and guilt. Given that the overt forms of aggression lead to the discharge of emotional tension, and the sensitivity has a small number of correlations with other emotional personality traits in general, we can conclude that healthy adolescents in the self-reports associate their emotional tension with a variety of manifestations of the emotional disadvantage (positive correlation).

EG adolescents associate their tension only with a sense of guilt, mental instability and its "psychoticism" component (intrapersonal problems), with an index of hostility and its "suspicion" component. Thus, there is a kind of "blind spot" related to the absence of a significant relationship to anxiety, neuroticism and depression. Given that the level of these indicators in EG is considerably higher (see. 3.1), and that in the literature they are considered as the main predictors of chronic somatic diseases, we can assume: adolescent patients are not fully aware of the emotional stress associated with manifestations of neuroticism, anxiety, depression. That could be one of the factors of chronicity.

There is another "blind spot" in the correlations with guilt. In addition to a positive correlation with emotional tension, which we have already mentioned, there is only a significant negative correlation with indirect aggression. In healthy adolescents guilt is significantly associated with a wide range of features (see. Table. 3).

On the one hand, the "blind spots" identified may represent the manifestations of alexithymia. Indeed, a number of studies confirm the role of alexithymia in the development of somatic problems (De Gucht et al., DOI:10.1016/j.paid. 2003.06.012/, 2004; Rieffe et al., DOI:10.1016/j.paid.2009.11.010, 2010). However, the work of F. C. Jellesma et al. allows not supporting this hypothesis (F.C. Jellesma et al., DOI: 10.1080/08870440801998970, 2009). These researchers also documented more pronounced alexithymia in adolescents with multiple somatic complaints, i.e. with somatoform disorders. However, they carried out a detailed study of their feelings through interviews. It was found that higher scores on alexithymia are explained by the difficulties the adolescents had in describing their negative internal states and in the experience of indefinable internal states, rather than difficulty in identifying emotions. These children reported higher intensities of fear and sadness. Their attention to the emotions, the ability to determine the causes of emotions were normal. Moreover, these teens better described their emotional experiences from the past and determined mixed emotions. Therefore, researchers believe that the teenagers had difficulties in processing their negative emotions, and not alexithymia in its conventional sense.

In our study, the hypothesis about the difficulties in the processing of negative emotions is supported by the results on irritability peculiar to the adolescent patients. The level of irritability is twice higher than in CG (see. 3.1). Furthermore, irritability in adolescent patients has a very wide range of correlations with other characteristics. In healthy adolescents as it is associated almost exclusively with open manifestations of aggression. Irritability is defined as an excessively active response of a human to subconscious to external and internal stimuli. Irritability is often peculiar to chronically ill adolescents (D.N. Isaev, 2005). Usually it is associated with manifestations of asthenia due to illness. However, from the perspective of psychological conditioning of somatic diseases, it can be assumed that the accumulated irritability reflects unconscious or not fully conscious emotional problems.

Thus, it is possible that due to certain reasons (increased sensitivity and vulnerability, greater intensity of experienced fear and sadness, parental ban to express negative emotions, etc.), such teenagers from an early age began to exclude particularly painful negative experiences of the current focus of consciousness. Children may feel periodically fully or partially repressed emotional distress as a vague, unpleasant, undifferentiated background, or as pointless annoyance, but the consciousness cannot effectively process them. If they are not clearly aware of the superseded negative emotions, they will not be able to feel guilt about it. As a result, blocked emotions supporting excitement in the subcortical areas of the brain, co-materialize through the hypothalamus and the autonomic nervous system, and maintain a disease of the visceral organs in a chronic state. Confirmation or refutation of the assumptions made here would be a future perspective of the present study.

\section{Conclusion}

In adolescents with chronic physical illness we observed the increased trait anxiety and mental instability, on these indicators they are significantly different from their healthy peers. They have much higher levels of neuroticism, sensitivity, slightly higher tendency to depressive symptoms. Differences in the level of emotional tension are closed to negligible. Indicators for various forms of aggressive reactions in both groups are similar and slightly increased, which is typical for the age group of adolescence. By the same time the hostility is more expressed, than the manifestation of overt aggression. The differences lie in the fact that in adolescents with chronic physical disease the irritation is much more pronounced. They recorded a higher level of indirect aggression, but on this indicator the differences are minimal and are closed to negligible. 
All of the surveyed adolescents reported extensive correlations of other emotional personality traits with trait anxiety and mental instability. The distinctive characteristics of the group of chronically ill adolescents are the extensive correlations with neuroticism and psychoticism. Perhaps this fact indicates a certain filter, when the background inclusion of negative emotions and their own intrapersonal problems mediate the perception.

A kind of "blind spot" has been discovered in the form of lack of correlations of emotional tension with neuroticism, trait anxiety and a tendency to depression in chronically ill adolescents. They are often referred to as the main predictors of somatization. In healthy adolescents, there is a wide range of emotional tension correlations with other characteristics. The second distinctive "blind spot" is the lack of correlation with guilt, excluding the relation with emotional tension and negative correlation with indirect aggression. Complements to this complex are extensive correlations of irritability with other traits.

This allows suggesting that adolescents have protective property to displace part of their negative emotions of the current focus of consciousness. Consequently, they cannot be efficiently recycled, and somatize supporting chronic disease.

\section{References}

Baranov, A.A., 2012. The health state of children in the Russian Federation. Pediatrics. Vol. 91. № 3. pp. 9-14.

Yenikolopov, S.N., 1990. Questionnaire of Bass-Darky / Workshop on psychodiagnostics. Psychodiagnostics of motivation and selfregulation. M.: MSU. pp 6-11.

Ilyin, E.P., 2002. Emotions and feelings. SPb.: Piter.

Isaev, D.N., 2005. Psychosomatic and somatopsychic disorders in children. SPb.: Speech.

Kazakov, I.A., 2009. Psychological diagnostics and correction of emotional disturbances in chronic gastritis. Ps.D thesis. SPb.

Kurtanova, Y.E., 2004. Personality characteristics of children with different chronic somatic diseases. Ps.D thesis. M. URL: http://psychlib.ru/inc/absid.php?absid=78515.

Malkina-Pikh, I.G., 2008. Psychosomatics: A Handbook of Practical Psychology. M.: Eksmo.

Malyarchuk, N.N., 2010. The values and meaning of life orientations of teachers with psychosomatic disorders. Bulletin of Applied Psychology of Education. № 3. pp. 36-42.

Marincheva, L.P., Zlokazova M.V., and Solovyov A.G., 2012. Features of etiopathogenesis of psychosomatic and somatoform disorders. Kazan Medical Journal. № 3. pp. 465-468.

Melnikov, V.M., and Yampolsky L.T., 1985. Introduction to experimental psychology of an individual. M: Education.

Pergamenschikov, L.A., Furmanov I.A., Aladin A.A., and Otchik S.V., 1992. Psychological diagnostics and therapy in the educational process. Minsk.

Frolov, O.V., 2009. Psychological characteristics of patients with chronic somatic diseases. Kazan pedagogical journal. № 9-10. pp. 104112.

Khanin, Y.L., 1976. Quick guide to the use of the scale of reactive and trait anxiety Charles D. Spielberger. L.

Kholmogorova, A.B., and Garanyan N.G., 1999. Culture, emotions and mental health. Psychology questions. № 2. pp 61-74.

Shmelev, A.G., 1988. Eysenck's Test / Workshop on psychodiagnostics. Psychodiagnostic materials. M.: MSU. pp 11-16.

De Gucht, V., Fischler B., and Heiser W., 2004. Neuroticism, alexithymia, negative affect, and positive affect as determinants of medically unexplained symptoms. Personality and Individual Differences, 36 (7), pp. 1655-1667. DOI:10.1016/j.paid.2003.06.012/

Efremova, G.I., Timoshenko G.V., Leonenko E.A., and Bochkovskaya I.A., 2014. Emotional experiences of person as a reason of psychosomatic risk origin. Asian Social Science, 10 (22), pp. 263-269. DOl:10.5539/ass.v10n22p263.

Ferro, M.A. and Boyle M.H., 2013. Longitudinal invariance of measurement and structure of global self-concept: a population-based study examining trajectories among adolescents with and without chronic illness. Journal of Pediatric Psychology, 38 (4), pp. 425-437. DOI:10.1093/jpepsy/jss112.

Halfon, N. and Newacheck P.W., 2010. Evolving notions of childhood chronic illness. Journal of the American Medical Association, 303, pp. 665-666. DOI:10.1001/jama.2010.130.

Howren, M.B. and Suls J., 2011.The symptom perception hypothesis revised: depression and anxiety play different roles in concurrent and retrospective physical symptom reporting. Journal of personality and social psychology, 100 (1), pp. 182-95. DOl:10.1037/a 0021715.

Jellesma, F.C., Rieffe C., Terwogt M.M., and Westenberg M., 2009. Do I feel sadness, fear or both? Comparing self-reported alexithymia and emotional task-performance in children with many or few somatic complaints. Psychology and health, 24 (8), pp. 881-893. DOI: $10.1080 / 08870440801998970$.

Pao, M., and Bosk A., 2011. Anxiety in medically ill children/adolescents. Depression and Anxiety, 28 (1), pp. 40-49. DOI:10.1002/da. 20727

Perrin, J.M., Bloom S.R., and Gortmaker, S.L., 2007. Increasing childhood chronic conditions in the United States. Journal of the American Medical Association, 297 (24), pp. 2755-2759. DOl:10.1001/jama.297.24.2755.

Pinquart, M. and Shen Y., 2011. Depressive symptoms in children and adolescents with chronic physical illness: an updated metaanalysis. Journal of Pediatric Psychology, 36 (4), pp. 375-384 DOI:10.1093/jpepsy/jsq104.

Pinquart, M. and Shen Y., 2011. Behavior problems in children and adolescents with chronic physical illness: a meta-analysis. Pediatric Psychology 36 (9): pp. 1003-1016. DOI:10.1093/jpepsy/jsr042. 
Rieffe, C., Oosterveld P., Meerum Terwogt M., Novin S., Nasiri H., and Latifian, M., 2010. Relationship between alexithymia, mood and internalizing symptoms in children and young adolescents: evidence from an Iranian sample. Personality and Individual Differences, 48 (4), pp. 425-430. DOI:10.1016/j.paid.2009.11.010.

Suris, J.C., Michaud P.A., and Viner R., 2004. The adolescent with a chronic condition. Part I: Developmental issues. Archive of Diseases in Childhood, 89, pp. 938-942. DOl:10.1136/adc.2003.045369.

Xie, H, Cairns R.B., and Cairns B.D., 2002. The development of social aggression and physical aggression: a narrative analysis of interpersonal conflicts. Aggressive Behavior, 28 (5), pp. 341-355. DOI:10.1002/ab.80008. 